\title{
Analysis and Study on the Handling Leakage Accident of Bulk Chemical Carriers Based on Bayesian Network
}

\author{
Wang Hong, Li Huabin
}

\begin{abstract}
On the basis of studying and studying a large number of literatures, this paper explores the types of navigation accidents of bulk carriers, focusing on self-sinking, grounding and collision accidents. This paper probes into the causes of bulk carrier navigation accidents from four aspects: human factors, ship factors, environmental factors and organizational factors. Through the investigation report of 58 accidents of cosmetics bulk carriers collected from the official websites of the Maritime Safety Administration, the network node was identified, the chain of accidents of bulk carriers was found and the Bayesian network of accidents of bulk carriers was constructed. Then the Bayesian network model was run, and 18 samples were selected to validate the model and confirm the feasibility of the model. Secondly, the types of dry bulk ships navigation accidents are analyzed to find out the types of frequent accidents. The most probable causal chain of collision, self-sinking and grounding of dry bulk carrier is obtained by analyzing the causal chain of these three accidents. Finally, the sensitivity of the Bayesian network is analyzed to determine the most likely cause of a ship accident. According to the research results, the countermeasures to prevent accidents are put forward and summarized. The research in this paper has certain guiding significance for relevant management departments to prevent the accidents of dry bulk carriers, and also provides a good guidance for shipping companies on the operation of dry bulk carriers, it provides a basis for the design, maintenance and operation of ships, and puts forward a good prediction and preventive measures.
\end{abstract}

Index terms- Ship Accident; Bayesian Network; Accident Type; chemical; cause analysis

\section{INTRODUCTION}

According to Shipsfocus, the volume of global trade in the transport of chemicals by sea rose 7.3 per cent to 196 million tonnes in 2017, well above the average growth rate of 2 per cent over the past five years and 3 per cent over the past decade. SHIPSFOCUS expects chemical trade volume to grow by a more modest 4 per cent in 2018 than the average. Intra-north-east Asia Route Intra-NEAsia was first with 26.9 $\mathrm{m}$ tonnes, up 6.9 per cent year-on-year, while intra-Continental Europe route Intra-UK Cont was second with $25.3 \mathrm{~m}$ tonnes, up 9 per cent. In third place was

Wang Hong; College of Naval Architecture and Ocean Engineering, Jiangsu University of Science and Technology, 2nd Road, Mengxi Road, Zhenjiang City, Jiangsu Province, China .Phone; 0511-84407400

Li Huabin: College of Naval Architecture and Ocean Engineering, Jiangsu University of Science and Technology, 2nd Road, Mengxi Road, Zhenjiang City, Jiangsu Province, China. Mobile:18796089295
Millde-East / Ne Asia, with total trade of 17.1 million tonnes, up 4.8 per cent year-on-year. The NAFTA-south American route soared 20 per cent to $6.7 \mathrm{~m}$ tonnes, making it into the top five. China accounts for 75 per cent of chemical trade and 70 per cent of net growth in north-east Asia's intraregional routes. By the end of June 2018, the global fleet of 4,322 liquefaction vessels was 103 million tons (less than 52,000 tons). More than 70 percent of them were built in China, Japan and South Korea. With the increase of the amount of chemicals transported by sea, the accidents of chemical leakage also happen constantly. According to Wikipedia's List of Shipwrecks, there were 643 ship accidents between 2012 and 2016 , with a total of 291 , or $45 \%$ of the total. The total number of accidents involving liquid cargo vessels was 55 , accounting for $8.59 \%$ of the total number of accidents in the past five years. Of the 291 accidents of the four main ship types in the past five years, the proportion of liquid cargo ship accidents is $18.21 \%$, second only to $64.26 \%$ of the general cargo ship, which is the ship type with more accidents. Of the 53 accidents, 18(33\%) were fire and explosion accidents caused by leakage at the wharf, which was the most frequent accident type, while 15(29\%) were stranded accidents, which was the second most frequent accident type Then there were 8 collision accidents $(15 \%), 5$ overturning accidents and 5 water ingress accidents (10\%), and 2 accidents with unknown causes $(2 \%)$. [1]

\section{RESEARCH CONTENT AND METHODOLOGY}

According to the complex and rigorous loading and unloading process of the chemical tanker, and based on the available risk assessment data of the chemical tanker, the leakage accident of the chemical tanker's loading and unloading operation was analyzed by using the Bayesian network. [2] The main research work is as follows:

(1) Through the search of a large number of chemical ship leakage accident sample data, and combined with a large number of related literature research, find out the human factors, equipment factors, environmental factors, cargo characteristics and management factors that lead to the leakage of the chemical tanker, and find out the causal relationship between the factors in the sample data. 
(2) Determine Bayesian network structure. By analyzing the complete causal chain of each incident, and then integrating all the single incident chains, a Bayesian network topology is drawn. Each factor leading to the accident is a node in the network, for each node to determine its definition and give the corresponding value range.

(3) Determine the conditional probability table of the network. According to the sample data of the chemical ship leakage accident, the node variables are assigned and the prior probability and the conditional probability table of each node are calculated. The conditional probability table is the mathematical basis of the reasoning operation of the Bayesian network, and is also a complicated step in constructing the Bayesian network model. This paper determines the conditional probability between parent node and child node based on leak sample data, Near Miss reports, relevant research results and expert score. The K-S test and the Mann-Whitney $U$ test are used to test the data given by experts with SPSS to ensure its reliability and objectivity. [3]The controllability of conditional probability is further improved.

(4) Build the Bayesian network model and use GeNIe or Hugin to simulate the model. From a specific accident through the collection of evidence input to the model, and then through model reasoning calculation, the accident probability. The selected samples are verified one by one.

(5) Analysis of sensitive factors and study of risk control measures: Because of the bidirectional inference characteristic of Bayesian networks, the sensitive factors that affect the risk of leakage in the loading and unloading of chemical vessels are found out through this characteristic, and reasonable suggestions are put forward for the sensitive factors, and forecast its risks. According to the risk factors, it provides the reference for taking the corresponding risk control measures, so as to improve the safety level of chemical loading and unloading. After the Bayesian Network topology is constructed, further Bayesian reasoning can be carried out. [4]

At present, there are two kinds of Bayesian reasoning: causal reasoning and diagnostic reasoning. Causal reasoning, that is, forward reasoning. [5]To get the probability of the outcome, we must determine the cause of the outcome, and the probability of the outcome deriving from the cause is Bayesian network reasoning. As shown in figure, the probability of occurrence of child node a is derived from parent nodes B and C. Diagnostic reasoning, also known as sensitivity analysis. If the occurrence probability of parent node $\mathrm{B}$ and $\mathrm{C}$ in the Bayesian network is increased to $100 \%$, the risk probability of child node a will change accordingly. The risk sensitivity of parent node $\mathrm{B}$ and $\mathrm{C}$ can be obtained by comparing the change value of a before and after, that is to find out the main cause of the result.

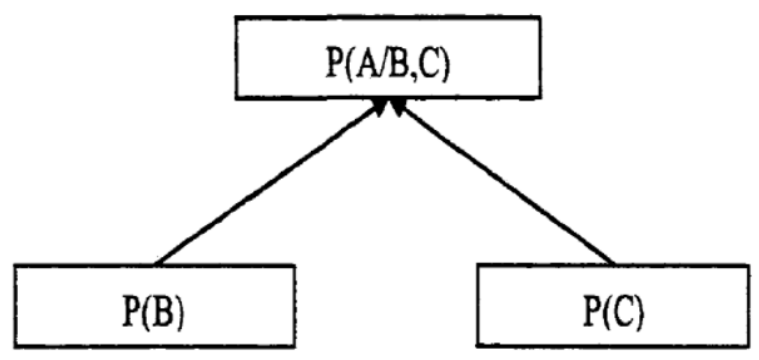

Figure 1Bayesian reasoning

2. Cause chain analysis of leakage accident in bulk chemical loading and unloading .Based on the theoretical analysis of the causal chain, the initial structure of the Bayesian Network of the chemical spill accident in bulk handling was obtained.

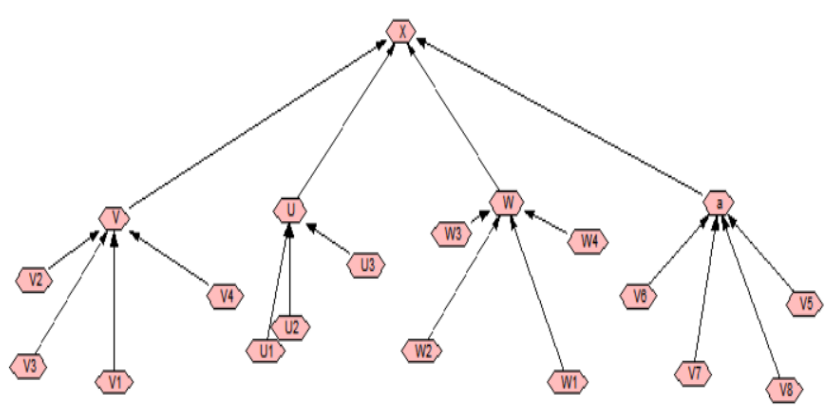

Figure 2 Bayesian network initial structure

Secondly, according to the expert opinion, the initial structure adjustment. The branch structure of environmental factors and ship factors is clear, and the optimization of the branch structure is mainly aimed at the artificial factors, and the MDL measure is used to calculate and compare the two 
structures, and the one with smaller total description length is chosen. In this article, the partial structure of the incomplete information (HA2) node and its parent node has been changed.

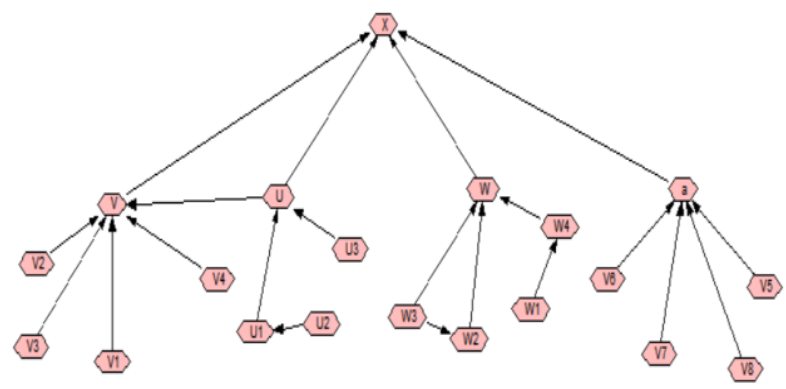

Figure 3 Constructing a new Bayesian network

The degree of dependency between Bayesian Network, known as conditional probability, is the basis of Bayesian network reasoning. In this paper, the determination of conditional probability table is based on the data in the accident samples by mathematical statistics. According to the knowledge of Bayes parameter learning, this section uses Bayes expectation type to estimate conditional probability.

It should be emphasized that all the conditional probabilities determined in this paper are based on the statistics from the samples of chemical handling and leakage accidents that have occurred, so all of the cause nodes listed here will eventually lead to a chemical spill. Although in the actual process of loading and unloading, the chemical loading and unloading leakage accident is extremely accidental, the probability is very small, but this paper studies the formation mechanism of the chemical loading and unloading leakage accident, it is based on the accident has occurred under the premise of the analysis of the study. [6]The resulting Bayesian network can be used to determine the most sensitive causal chain of the accident, as well as to analyze the effects of various factors on the crash after the crash, and to determine the "1" state of some factor nodes, possibility of collision of ships. In this paper, 28 container ship collision accidents have been collected and 18 samples have been obtained. [7] The conditional probability table is used as an example.

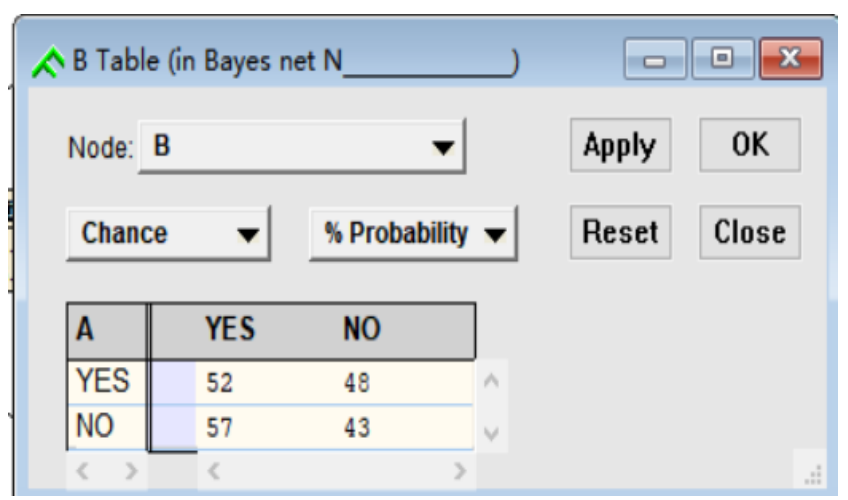

Figure 4 Netica setup diagram for human factors (low security awareness) node

III. PROSPECTS FOR LEAKAGE ACCIDENT OF BULK CHEMICAL CARRIERS BASED ON BAYESIAN NETWORK

Based on the analysis of the Bayesian network model of the chemical vessel leakage accident, the risk factors in the aspects of human factors, equipment factors, environmental factors, cargo characteristics and management factors are found, and the causes that are most likely to lead to the leakage accident are obtained, the corresponding preventive measures are put forward according to the reasons. This has certain guiding significance to the prevention of chemical ship leakage accident for crew and shipping company, and also has certain reference value to the competent authority.

\section{IV.CONCLUSION}

The leakage from chemical tanker is a complicated and changeable system, and it is difficult to quantify and study it accurately by General Network because of the diversity of the factors leading to the accident and the interrelation among them, the use of Bayesian network can be a good solution to this problem, resulting in reduced fruit deviation due to mold and quantification. Because of the Bayesian Network's ability to deal with uncertain events, it is scientifically valid to use it for causal analysis in ship systems. The BAYESIAN network is also a network where new accident samples can be added for training and learning, and the more data there is, the more accurate the training results will be, therefore, the use of Bayesian Network for Causal Analysis and Risk Analysis and prediction of accidents is worthy of promotion. At present, the research on Bayesian network is becoming more and 
more deep, and the algorithms used in Bayesian network are becoming more and more mature and scientific. Researchers have developed more Bayesian network software that makes it faster and easier to use Bayesian reasoning. [8] Such as Hugin, GeNIe, Bayesia Lab, Matlab-BNT, Bayes Builder, Netica and other Bayesian software.

\section{REFERENCES}

[1]Ozcan Arslan,Ismail Deha Er. SWOT analysis for safer carriage of bulk liquid chemicals in tankers [J]. Journal of Hazardous Materials, 2007, 154(1).

[2]George Psarros, Rolf Skjong,Erik Vanem. Risk acceptance criterion for tanker oil spill risk reduction measures[J]. Marine Pollution Bulletin,2011,62(1).

[3]Emre Akyuz,Metin Celik. A hybrid human error probability determination approach: The case of cargo loading operation in oil/chemical tanker ship[J]. Journal of Loss Prevention in the Process Industries,2016,43.

[4]S. Bonvicini,P. Leonelli,G. Spadoni. Risk analysis of hazardous materials transportation: evaluating uncertainty by means of fuzzy logic[J]. Journal of Hazardous Materials, 1998,62(1).

[5]Robin K.S. Hankin. Major hazard risk assessment over non-flat terrain. Part I: continuous releases [J]. Atmospheric Environment,2003,38(5).

[6]Beatriz Molina Serrano,Nicoleta González-Cancelas,Francisco Soler-Flores,Alberto Camarero-Orive. Classification and prediction of port variables using Bayesian Networks[J]. Transport Policy,2018,67.

[7]Major Hazard Aspects of the Transport of Dangerous Substance,Health and Safety. ACDS. . 2016

[8]French D P,Howlett E,Mendelsohn D. Oil and Chemical Spill Impact Model System Description and Application. Proceedings Seventeenth Arctic and Marine Oil Spill PrograTechnical Seminar . 1994

Wang Hong ; Professor, Graduate Instructor, School of Naval Architecture and Ocean Engineering, Jiangsu University of Science and Technology.

Li Huabin ; Master student, chief mate for ocean-going ships. 